\title{
miRNA-299-5p regulates estrogen receptor alpha and inhibits migration and invasion of papillary thyroid cancer cell
}

This article was published in the following Dove Press journal: Cancer Management and Research

\section{Zhihong Wang \\ Liang $\mathrm{He}$ \\ Wei Sun \\ Yuan Qin \\ Wenwu Dong \\ Ting Zhang \\ Ping Zhang \\ Hao Zhang}

Department of Thyroid Surgery, The First Hospital of China Medical University, Shenyang II000 I, China
Correspondence: Hao Zhang

Department of Thyroid Surgery,

The First Hospital of China Medical

University, No 155, Nanjing Street,

Heping District Shenyang, Liaoning

Province, II000I, China

Tel +862483282191

Email haozhang@cmu.edu.cn
Background: The incidence of papillary thyroid cancer (PTC) is increasing faster than any other solid tumors worldwide. Invasion and metastasis are the main reasons for the poor prognosis of patients with PTC. Previously, we observed significantly low expression of miRNA-299-5p in invasive PTC tissue samples.

Aim: The present study aimed to determine whether miR-299-5p plays a key role in PTC migration and invasion.

Materials and methods: The miR-299-5p expression level was measured using quantitative real-time PCR in 109 human PTC samples and paired adjacent normal tissues and in the human BCPAP PTC cell line. The effects of miR-299-5p on PTC cell migration and invasion were assessed using wound healing and transwell assays. In addition, we searched for the miR-299-5p target, and the potential mechanism was demonstrated using a reporter assay and rescue experiment.

Results: The expression of miR-299-5p was associated with gender and extrathyroidal extension, and an elevated level of miR-299-5p suppressed BCPAP cell migration and invasion. Estrogen receptor $\alpha(E R \alpha)$ is a direct target of miR-299-5p. The expression level of ER $\alpha$ was significantly higher in PTC tissues and was associated with migration and invasion in PTC cells. Overexpression of ER $\alpha$ could impair miR-299-5p-induced inhibition of migration and invasion. As a key factor of the pathway related to PTC invasion, Gli1 can be combined with ER $\alpha$ and can be regulated by miR-299-5p.

Conclusion: Our data suggested that miR-299-5p could participate in PTC migration and invasion and could be a potential therapeutic target for patients with aggressive PTC tumors.

Keywords: miR-299-5p, papillary thyroid cancer, PTC, estrogen receptor $\alpha$, ER $\alpha$, migration, invasion

\section{Introduction}

Papillary thyroid cancer (PTC), comprising more than $80 \%$ of all thyroid carcinomas, is the prevalent histotype of thyroid cancer. ${ }^{1}$ It is usually a slow-growing cancer, with a good prognosis and therapeutic response. Although the overall survival from PTC is $97 \%$, patients with advanced PTC have a 5-year survival rate of only $59 \%$. $^{2}$ Lymph node metastasis (LNM), extrathyroidal extension (ETE), and distant metastasis are associated with advanced tumor node metastasis (TNM) stages and poor prognosis. ${ }^{3}$ Therefore, efforts should be made to improve the prognosis of these patients with aggressive tumors.

Accumulating evidence suggests that miRNAs are involved in many important physiological and pathological processes, such as cell differentiation, development, and proliferation. miRNAs are widely dysregulated in different cancers including thyroid cancer. ${ }^{4,5}$ Our previous microarray analysis study showed that miR-299-5p was 
significantly downregulated in ETE PTC tissues compared with that in non-ETE PTC tissues. ${ }^{6}$ miR-299-5p is also significantly downregulated and can act as a tumor suppressor in various tumors, including prostate cancer, breast cancer, hepatocellular cancer, and colorectal cancer. ${ }^{7-10}$ However, the expression, clinicopathological significance, and function of miR-299-5p in PTC require further investigation.

The reason for high incidence of PTC in women is unclear. Studies have revealed that the estrogen receptor (ER), especially $\mathrm{ER} \alpha$, plays an important role in the development and progression of PTC. ${ }^{11,12}$ Upregulation of ER $\alpha$ expression in PTC cells increased the activity and secretion of MMP-2 and MMP-9 and promoted cell invasion and metastasis. ${ }^{13}$ Our previous study indicated that the expression of ER $\alpha$ was significantly associated with gender and ETE in PTC tissues. Moreover, ER $\alpha$ can promote the invasion of the ER $\alpha$-positive PTC cell line BCPAP when treated with the ER $\alpha$-selective agonist 1,3,5-tris (4-hydroxyphenyl)-4-propyl-1H-pyrazole (PPT). ${ }^{14,15}$

The transcription factor Gli1 is both a downstream effector of the Hedgehog (Hh) pathway and a target protein regulated by this pathway, and its expression is a hallmark of activation of the Hh pathway. ${ }^{16}$ Some studies suggest the relationship between Glil expression and tumor invasion and metastasis. ${ }^{17,18}$ Our previous study showed that the expression of Gli1 in the Hh signaling pathway in PTC tissues was significantly higher than that in normal tissues, and its expression level correlated with tumor size, extrathyroidal invasion, and LNM. ${ }^{19}$ Studies on breast cancer have shown that overexpression of ER $\alpha$ in ER-positive breast cancer cells can upregulate the transcriptional activity of Gli1 and even promote the effect of Gli1 protein on cell invasion and metastasis. $^{20,21}$

We propose the following hypothesis: in ER $\alpha$-positive cell lines, miR-299-5 p may inhibit the invasion of PTC cells by targeting ER $\alpha$ to affect the expression of Gli1.

\section{Materials and methods}

A total of 109 human PTC tissues and 109 paired adjacent normal thyroid tissues were obtained from patients undergoing curative-intent surgery in Department of Thyroid Surgery, the First Hospital of China Medical University (Shenyang, China) between 2010 and 2014. All tissues were immediately frozen in liquid nitrogen and stored at $-80^{\circ} \mathrm{C}$ until processing. The inclusion criteria were as follows: all patients received initial surgery and none of the patients in our group had undergone prior oncological surgery or head and neck irradiation. Clinicopathological information, such as tumor size, the presence of ETE, and LNMs, were collected from the relevant medical records. ETE was defined as invasion of an adjacent tissue or an organ or the skeletal muscle outside the isthmus. ${ }^{22}$ Our study was approved by the ethics committee of the First Hospital of China Medical University, Shenyang, China. Written informed consent was obtained from all study participants.

\section{Cell culture and transfection}

The ER $\alpha$-positive human PTC cell line, BCPAP, was bought from the Stem Cell Bank, Chinese Academy of Sciences (Shanghai, China) and maintained in Roswell Park Memorial Institute (RPMI) 1640 medium supplemented with 10\% FBS (RPMI 1640+10\% FBS+1\% nonessential amino acids $+1 \%$ sodium pyruvate+1\% Glutamax). ${ }^{23,24}$ The miR-299-5p mimic, inhibitor, and their respective negative control (NC) were purchased from Gene Pharma (Shanghai, China). siRNAs to silence ER $\alpha$ were designed and synthesized by Gene Pharma. The oligonucleotide sequences were as follows: miR-299-5p mimic (sense): 5'-UGGUUUACCGUCCCACAUACAU-3', NC (sense): 5'-UUCUUCGAACGUGUCACGUTT-3'. ER $\alpha$-siRNAs sequences used were 5'-CGAGUAUGAUCCUACCAGATT-3' (sense); the following nonsense siRNA was used as a NC: 5'-UUCUCCGAACGUGUCACGUTT-3' (sense). Gli1-siRNAs sequences used were 5'-AACUCCACAGGCAUACAGGAU-3' (sense); NC: 5' -AACGUACGCGGAAUACAACGA-3' (sense). Lipofectamine 2000 was used for transfection (Thermo Fisher Scientific, Waltham, MA, USA). The PTC cell line treated with the transfection reagent alone was used as a mock control. The normal human thyroid follicular epithelial cell line, Nthy-ori 3-1, was purchased from the European Collection of Authenticated Cell Culture (ECACC, UK).

Lentivirus and its NC were designed and synthesized by Genechem (Obio Technology Corp., Ltd., Shanghai, China). A total of $3 \times 10^{5}$ cells $/ \mathrm{mL}$ were seeded in each well of a six-well plates and then infected with pLenti-EF1a-EGFPP2A-Puro-CMV-ESR1-3Flag (vector-ER $\alpha$ ) or plenti-CMV3FLAG-GLI1-PGK-Puro (vector-Gli1), empty vector (EV; NC group), and no infection (nontransfected control group) by replacing the medium with infection medium containing recombinant vectors at a multiplicity of infection (MOI) of 40 plaque forming units per cell and $10 \mu \mathrm{L}$ of $1 \mathrm{mg} / \mathrm{mL}$ polybrene (Obio Technology Corp., Ltd.). Plates were then incubated for 24 hours before their media were changed to fresh, virus-free media. Cells were treated with puromycin ( $1 \mu \mathrm{g} / \mathrm{mL}$; InvivoGen, San Diego, CA, USA) to produce stable transfected cells (vector-ER $\alpha$, vector-Gli1, and EV) for further experiments. 


\section{Total RNA extraction and quantitative real-time reverse transcription PCR ( $q R T$ - PCR) detection}

Total RNA was isolated using RNAiso Plus (Takara Biotechnology Co., Ltd., Dalian, China). The 638315 Mir- $\mathrm{X}^{\mathrm{TM}}$ miRNA First-Strand Synthesis Kit (Takara Biotechnology Co., Ltd.) was used to transcribe total RNA to cDNA. qRT-PCR was performed using SYBR Premix Ex Taq II (Takara Biotechnology Co., Ltd.) on the LightCycler 480 system (Roche Diagnostics, Indianapolis, IN, USA). The qRT-PCR cycling profile was as follows: $95^{\circ} \mathrm{C}$ for 30 seconds, followed by 40 cycles at $95^{\circ} \mathrm{C}$ for 5 seconds and $60^{\circ} \mathrm{C}$ for 30 seconds. GAPDH and U6 were used as references for mRNAs and miRNAs, respectively.

\section{Cell migration and invasion assays}

Cell migration was evaluated using a wound-healing assay. After 24 hours of transfection, $4 \times 10^{5}$ cells were cultured in six-well plates until the cell confluence reached $90 \%$, and scratches were created by scraping the cell layer across each well using a $200 \mu \mathrm{L}$ pipette tip. Wound healing was observed under a light microscope, and images in three observation fields were captured at 0 hour and 24 hours. Transwell invasion assays were performed to determine cell invasion. After 24 hours of transfection, the cells were trypsinized, the cells were suspended into single cells in a serum-free medium, and the concentration of each group was adjusted to $4 \times 10^{4}$ cells $/ \mathrm{mL}$ using a serum-free medium. Cell invasion assays in vitro were performed using a Transwell chamber (Costar, Corning, NY, USA) with a polycarbonic membrane $(6.5 \mathrm{~mm}$ in diameter and $8 \mu \mathrm{m}$ pore size). Cells were incubated for 36 hours for the invasion assay. Afterward cells that had invaded to the lower surface of the membrane were fixed with $4 \%$ paraformaldehyde and stained with $0.5 \%$ crystal violet and washed twice with PBS. Stained cells were observed under an inverted microscope to count the cell number within five randomly chosen fields at $100 \times$ magnification, and the average number was calculated.

\section{Luciferase reporter assay}

The 3' UTR from ESR I (encoding ER $\alpha$ ) (ESR1-3'UTR [Wt]) and mutant ESR 1-3'UTR (Mut) containing the putative binding site of miR-299-5p were generated by PCR amplification, established, and cloned into the firefly luciferase expressing vector pmirGLO (Promega Corporation, Fitchburg, WI, USA) for a dual luciferase reporter assays. The $293 \mathrm{~T}$ cell line was purchased from the Stem Cell Bank, Chinese Academy of Sciences (Shanghai, China) and used for luciferase reporter assays. $293 \mathrm{~T}$ cells were plated into 24-well plates and cotransfected with $500 \mathrm{ng}$ of the ESRI-Wt or ESRl-Mut reporter vector and $100 \mathrm{nM}$ miR-299-5p mimics or $200 \mathrm{nM}$ of inhibitor using Lipofectamine 2000. After 48 hours, the cells were harvested, and luciferase activity levels were measured using a Dual-Luciferase Reporter Assay System (Promega Corporation) according to the manufacturer's protocol.

\section{Immunohistochemical analysis}

Twenty formalin-stabilized PTC tissues and adjacent normal thyroid tissues were embedded in paraffin and cut into $4 \mu \mathrm{m}$ sections for use in immunohistochemistry (IHC). Slides were deparaffinized, rehydrated, and subjected to microwave heat antigen retrieval in $0.01 \mathrm{~mol} / \mathrm{L}$ sodium citrate buffer ( $\mathrm{pH} 6.0$ ) for 20-25 minutes. After blocking endogenous peroxidase activity after 30 minutes at $37^{\circ} \mathrm{C}$, the sections were incubated with primary antibodies against $\mathrm{ER} \alpha$ (1:200; Abcam, Cambridge, UK) overnight at $4^{\circ} \mathrm{C}$. Immunoreactivity was visualized using the chromogen 3, 3'-diamino-benzidine (DAB; Maixin Bio, Fuzhou, China). The slides were then counterstained with hematoxylin, washed, dehydrated with alcohol and xylene, and mounted onto cover slips.

\section{Western blotting analysis}

Proteins were extracted from BCPAP cells and PTC and adjacent normal tissue samples from eight patients (four male and four female patients, and four patients were $<45$ years old and four were $\geq 45$ years old). The protein extracts $(20-30 \mu \mathrm{g})$ were separated on $10 \%$ SDS-PAGE gels and then electrophoretically transferred to polyvinylidene difluoride membranes (EMD Millipore, Billerica, MA, USA). Membranes were blocked in 5\% skim milk, which was dissolved in Tris-buffered saline with $0.1 \%$ Tween-20 (TBS-T) for 2 hours. The membranes were then incubated with primary antibodies for ER $\alpha$ (1:300; Santa Cruz Biotechnology Inc., Dallas, TX, USA), Gli1 (1:100; Santa Cruz Biotechnology Inc.), and GAPDH (1:3,000; Santa Cruz Biotechnology Inc.) overnight at $4^{\circ} \mathrm{C}$. The membranes were then washed three times and incubated with a 1:10,000 dilution of horseradish peroxidase-conjugated anti-rabbit or anti-mouse secondary antibody and visualized using enhanced chemiluminescence (ECL; Thermo Fisher Scientific).

\section{Coimmunoprecipitation}

For coimmunoprecipitation of Gli1 with ER $\alpha$, the following lysis buffer was used: $20 \mathrm{mM}$ Tris (pH 7.9), $150 \mathrm{mM} \mathrm{NaCl}$, $1 \mathrm{mM}$ EDTA, $0.3 \%$ Triton-X-100, proteinase inhibitor cocktail (Roche Applied Science, Penzberg, Germany), and halt phosphatase inhibitor cocktail (Thermo Fisher Scientific). 
The cell lysates were subjected to immunoprecipitation with anti-Gli1 antibodies (Santa Cruz Biotechnology Inc.) or antiER $\alpha$ antibodies (Santa Cruz Biotechnology Inc.). The eluates were separated by SDS-PAGE, and Western blotting analysis was performed using the anti-ER $\alpha$ antibodies $(1: 1,000)$ and anti-Gli1 antibodies $(1: 1,000)$.

\section{Statistical analyses}

All statistical analyses were performed using SPSS Statistics (version 20.0; IBM Corporation, Armonk, NY, USA) and GraphPad Prism 6 software (GraphPad Software, Inc., La Jolla, CA, USA). The results are presented as mean \pm SD and median (IQR). The relative expression levels of mRNAs or miRNAs were calculated using the $2^{-\Delta \mathrm{Ct}}$ method (cycle threshold [Ct]). The Mann-Whitney $U$ test, Student's $t$-test, and ANOVA tests were used to compare data from independent groups. Bonferroni correction was used to counteract the problem of multiple comparisons. The chi-squared test was applied to examine the relationship between miR-299-5p expression and the clinicopathological characteristics. $P$-value $<0.05$ was considered statistically significant.

\section{Results}

\section{miR-299-5p was downregulated in PTC tissues and cell lines and was associated with gender and ETE}

The expression of miR-299-5p was significantly lower in human PTC tissues $(n=109)$ compared with that in paired adjacent normal thyroid tissues (Figure $1 \mathrm{~A} ; P<0.001$ ). To further investigate the clinical significance of miR-299-5p, we examined the correlations between miR-299-5p expression and the clinicopathological features in PTC tissues. As shown in Table 1, there were significant differences in the
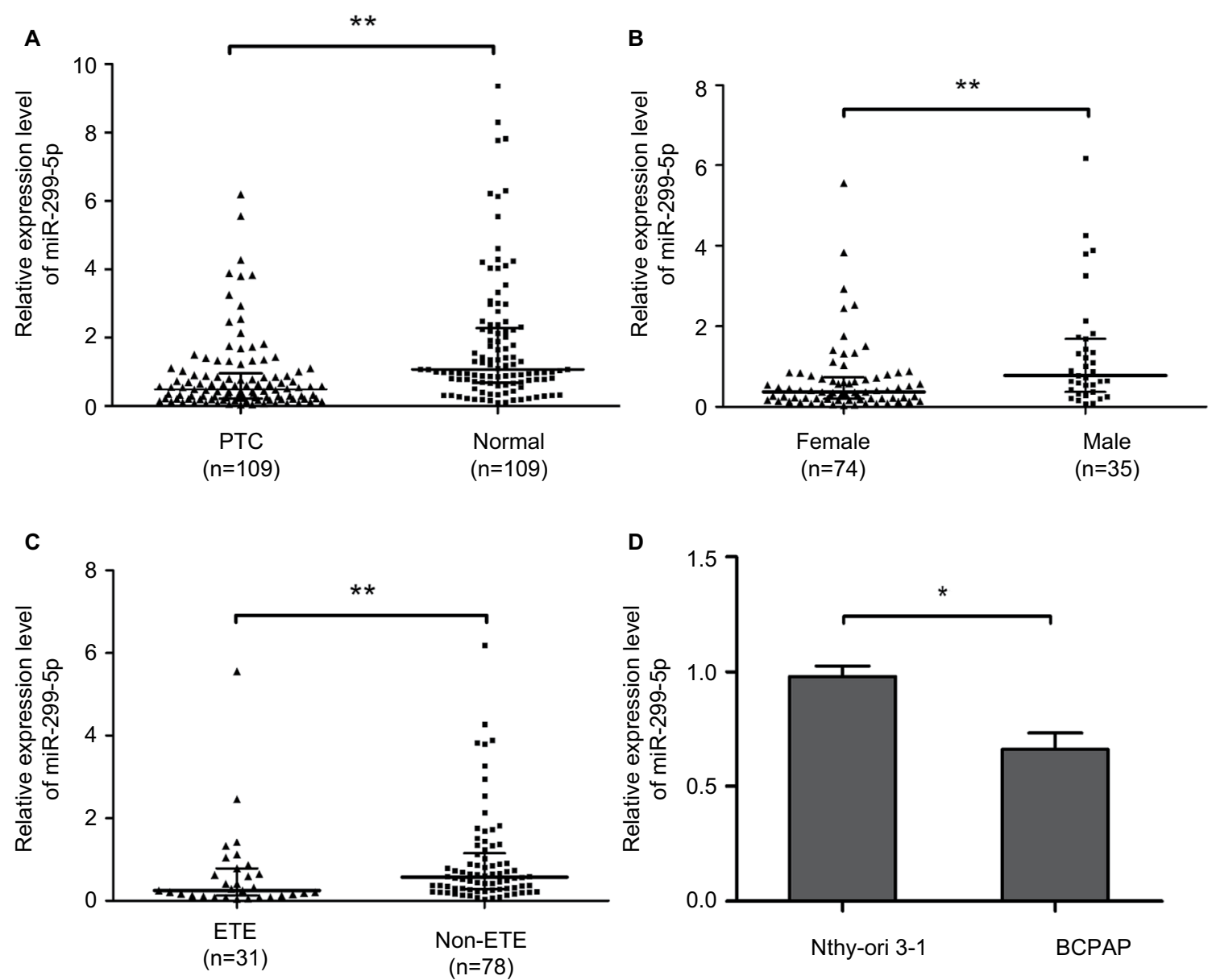

Figure I miR-299-5 p was downregulated and associated with gender and ETE in PTC tissues.

Notes: (A) The relative expression levels of miR-299-5p in 109 PTC tissues and paired adjacent normal thyroid tissues. (B) The relative expression levels of miR-299-5p in 74 female and 35 male PTC tissues. (C) The relative expression levels of miR-299-5p in 3 I ETE and 78 non-ETE PTC tissues. (D) The relative expression levels of miR-299-5p in PTC cell lines (BCPAP) and normal human thyroid follicular epithelial cell line (Nthy-ori 3-I). All experiments were determined by qRT-PCR. The Mann-Whitney $U$ test was used to analyze the differences between the two groups; data are presented as the median (IQR). ${ }^{*} P<0.05$; ${ }^{* *} P<0.0$ I.

Abbreviations: ETE, extrathyroidal extension; PTC, papillary thyroid cancer; qRT-PCR, quantitative real-time reverse transcription PCR. 
Table I Correlation of miR-299-5p expression with clinicopathologic characteristics in PTC

\begin{tabular}{|l|l|l|l|}
\hline $\begin{array}{l}\text { Clinicopathologic } \\
\text { parameters }\end{array}$ & $\begin{array}{l}\text { Case } \\
\text { number, } \\
\mathbf{n}(\%)\end{array}$ & $\begin{array}{l}\text { Relative } \\
\text { miR-299-5p } \\
\text { expression }\end{array}$ & P-value \\
\hline Total cases & 109 & & \\
\hline Age (years) & & & \\
\hline$\leq 45$ & $68(62.39)$ & $0.43(0.2 I-0.86)$ & 0.405 \\
\hline$>45$ & $4 I(37.6 \mathrm{I})$ & $0.64(0.24-1.12)$ & \\
\hline Gender & & & \\
\hline Male & $35(32.1 \mathrm{I})$ & $0.79(0.37-1.69)$ & $0.00 \mathrm{I}^{\mathrm{a}}$ \\
\hline Female & $74(67.89)$ & $0.37(0.20-0.74)$ & \\
\hline Cancer size (cm) & & & \\
\hline$\leq 4$ & $83(76.15)$ & $0.70(0.2 \mathrm{I}-1.34)$ & $0.36 \mathrm{I}$ \\
\hline$>4$ & $26(23.85)$ & $0.46(0.22-0.86)$ & \\
\hline Lymph node metastasis & & & \\
\hline Positive & $43(39.45)$ & $0.37(0.19-1.04)$ & 0.229 \\
\hline Negative & $66(60.55)$ & $0.57(0.27-0.94)$ & \\
\hline Extrathyroid extension & & & \\
\hline Positive & $31(28.44)$ & $0.25(0.13-0.78)$ & $0.0 I^{\text {a }}$ \\
\hline Negative & $78(7 I .56)$ & $0.57(0.28-1.15)$ & \\
\hline
\end{tabular}

Notes: All data were calculated using Mann-Whitney $U$ test. The data are represented as median (IQR). ${ }^{a}$ Statistically significant $(P<0.05)$.

Abbreviation: PTC, papillary thyroid cancer.

expression levels of miR-299-5p (relative quantification value) with regard to gender ( 74 females vs 35 males; Figure 1B; $P=0.0011)$. We compared the miR-299-5p expression between the same number of male and female samples ( $\mathrm{n}=35$; Figure S1). The estrogen levels get lower with decreased ovulation in women older than 45 years. Thus, the analysis of ER $\alpha$ expressions in PTC was performed in those patients of reproductive age ( $\leq 45$ years old) and advanced reproductive age ( $>45$ years old). We provided the differences in miR299-5p expression levels in males and females in different age subgroups. In female group, miR-299-5p expression was significantly lower in patients aged 45 years or younger than that in patients older than 45 years. The opposite result was found in the male group (Figure S2A and B). In addition, miR-299-5p was strongly associated with ETE in PTC (31 ETE vs 78 non-ETE; Figure $1 C ; P=0.01$ ), which was consistent with the previous microarray data. We detected miR-299-5p expression in ETE vs non-ETE patients in gender-specific and age-specific manners (Figure S3A-D). The patients with ETE included eight with perithyroid muscle invasion, 11 with recurrent laryngeal nerve, three with trachea, two with esophagus, five with carotid artery sheath, and two with jugular vein. miR-299-5p was downregulated in PTC cell lines (BCPAP) compared with that in normal human thyroid follicular epithelial cell line (Nthy-ori 3-1; Figure 1D; $P<0.05)$. miR-299-5p inhibited the migration and invasion of PTC cells
A wound healing assay and a transwell assay were used to assess the effect of miR-299-5p on migration and invasion, respectively, of PC cells in vitro. The results showed that miR-299-5p overexpression reduced the migration ability of BCPAP cells compared with that of the NC (Figure 2A; $P<0.001)$. Moreover, as shown in Figure 2B, the Transwell assays indicated that overexpression of miR-299-5p decreased the invasion abilities of BCPAP cells $(P=0.002)$. Taken together, these data indicated that miR-299-5p could inhibit migration and invasion in BCPAP cells.

\section{$E R \alpha$ is a direct target of miR-299-5p}

To investigate the mechanism of miR-299-5p in PTC, we screened for target genes of miR-299-5p using the target prediction tools such as TargetScan (http://www.targets can.org) and miRanda (http://www.microrna.org). Both prediction tools indicated that ESRI (encoding ER $\alpha$ ) could be a direct target of miR-299-5p. As predicted, we observed that overexpression of miR-299-5p decreased the expression of ER $\alpha$ at both mRNA and protein levels in BCPAP cells (Figure 3A). To assess whether miR-299-5p could directly bind to the 3'-UTR of ESR1 mRNA, the wild-type ESR1-3'UTR (Wt) and mutant ESR1-3'UTR (Mt) containing the putative binding site of miR-299-5p were established and cloned into a luciferase expressing vector. The results demonstrated that overexpression of miR-299-5p significantly suppressed the luciferase activity of the wild-type ESR1-3'UTR but failed to affect the mutant ESR1-3'UTR (Figure 3B). These data indicated that miR-299-5p could directly bind to the $3^{\prime}$-UTR of ESR1.

\section{ER $\alpha$ was overexpressed and associated with migration and invasion in PTC}

To further evaluate the effect of ER $\alpha$ on PTC development, we detected ER $\alpha$ expression in PTC using qRT-PCR, Western blotting, and IHC. PCR analysis revealed that ESR1 levels were significantly upregulated in PTC tissues $(\mathrm{n}=109)$ compared with those in paired adjacent normal thyroid tissues (Figure $4 \mathrm{~A} ; P=0.003$ ). The expression of ESR1 was significantly higher in the female group than in the male group (Figure 4B; $P=0.009$ ) and in the ETE group compared with that in the non-ETE group (Figure $4 \mathrm{C} ; P=0.01$ ), which was consistent with our previous work. We compared the ESR1 expression between same number of male and female samples $(\mathrm{n}=35)$. The results still showed that ESR1 expression in the female group was significantly higher than that in the male group (Figure S4). The IHC results showed strong positive staining for ER $\alpha$ in PTC tissues, whereas staining was absent 
A

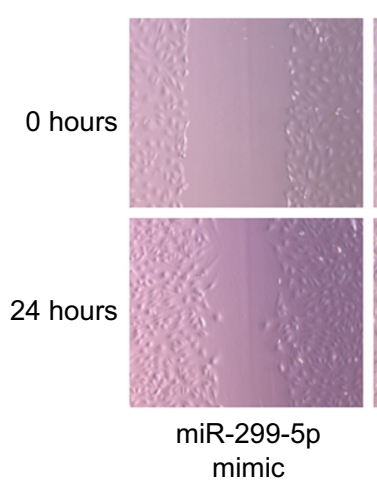

B

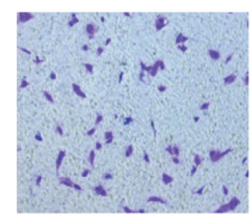

miR-299-5p mimic

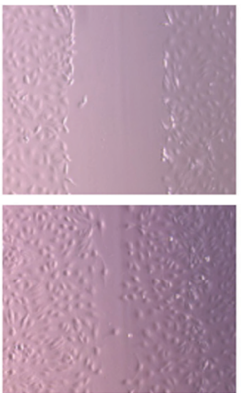

NC

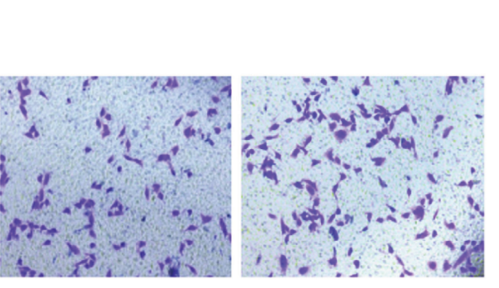

NC

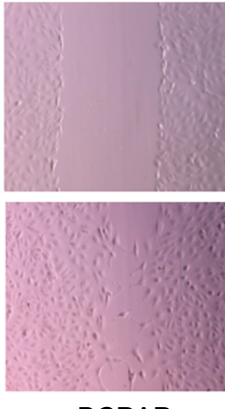

BCPAP

BCPAP

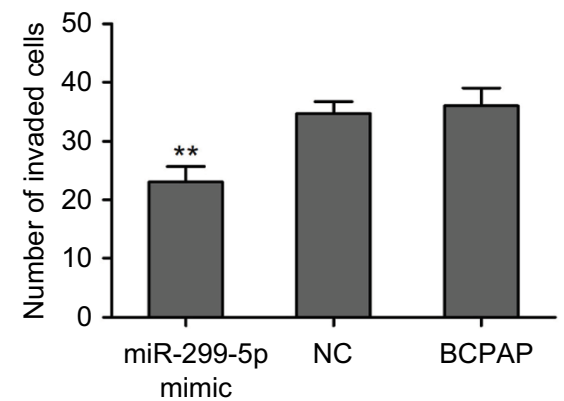

Figure 2 miR-299-5p inhibited the migration and invasion of PTC cells.

Notes: (A) A wound healing assay was applied to analyze the migration capacity of PTC cells after transfection with miR-299-5p mimic or NC. (B) Transwell assays were used to evaluate the invasion capacity in PTC cells after transfection with miR-299-5p mimic or NC. For the migration and transwell assays, cells in five random fields were counted under a microscope at $100 \times$ magnification. Data are presented as the mean $\pm S D$. $* * P<0.01$ vs NC.

Abbreviations: NC, negative control; PTC, papillary thyroid cancer.

or sporadic in adjacent normal thyroid tissues (Figure 4D). Moreover, Western blotting analysis of samples from eight patients also revealed that the level of ER $\alpha$ in PTC tissues was higher than that in adjacent normal thyroid tissues (Figure 4E). We demonstrated that overexpression of ER $\alpha$ increased the invasion abilities of BCPAP cells $(P=0.002)$, while inhibiting $\mathrm{ER} \alpha$ expression decreased the invasion abilities, as assessed using transwell assays $(P=0.0011$; Figure $4 \mathrm{~F})$. These results demonstrated that ER $\alpha$ was overexpressed and associated with migration and invasion in PTC.

\section{Overexpression of ER $\alpha$ could impair the miR-299-5p-induced inhibition of migration and invasion}

Next, we adapted a "rescue" strategy to further confirm whether the inhibition of migration and invasion effects of miR-299-5p was mediated via ER $\alpha$. The vector-ER $\alpha$ was first transfected into BCPAP cells to construct a stable ER $\alpha$ overexpression BCPAP cell line. The PCR and Western blotting results indicated that overexpression of $E R \alpha$ significantly rescued the inhibition of $E R \alpha$ expression in the presence of miR-299-5p mimic (Figure 5A). Wound healing assays showed that overexpression of ER $\alpha$ significantly rescued the migration inhibition role of miR-299-5p (Figure 5B). Transwell assays showed that overexpression of ER $\alpha$ significantly attenuated inhibition of the invasion induced by miR-299-5p (Figure 5C). These results demonstrated that miR-299-5p inhibited migration and invasion by targeting ER $\alpha$ in BCPAP cells.

\section{miR-299-5p regulated migration and invasion through the ER $\alpha / G$ lil complex}

To better understand the underlying molecular mechanism of miR-299-5p-mediated inhibited migration and invasion of PTC, we detected the Glil protein that is associated with the ER $\alpha$ pathway. IHC revealed that the protein levels of Gli1 were strongly and positively stained in PTC tissues (Figure 6A). Western blotting results showed that the protein level of Gli1 in PTC tissues was higher than that in adjacent normal thyroid tissues (Figure 6B). Overexpression of Gli1 increased the invasion abilities of BCPAP cells $(P=0.002)$, while inhibiting Glil expression decreased their invasion abilities, as assessed using Transwell assays $(P=0.03$; Figure 6C). Therefore, we hypothesized that 
A

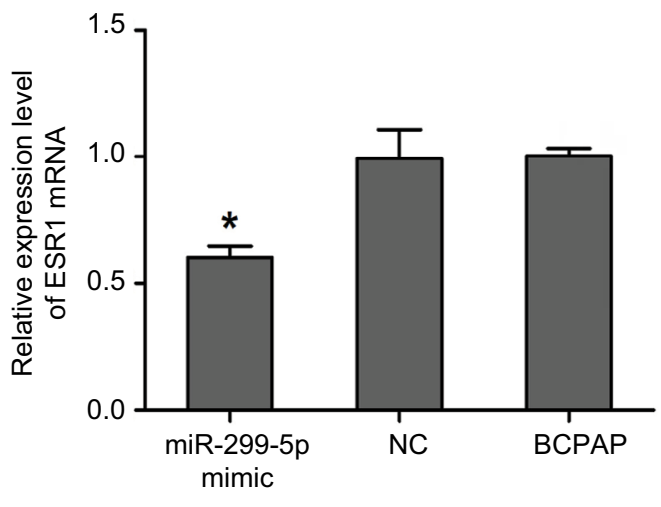

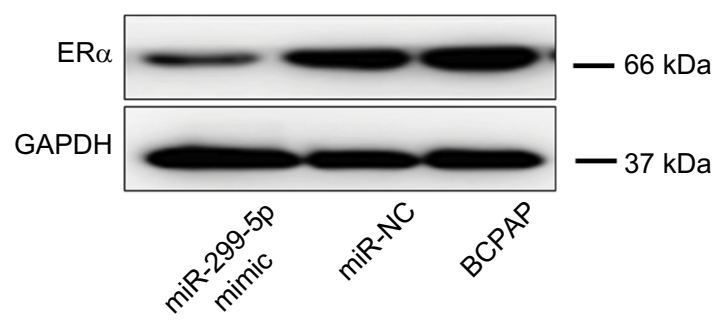

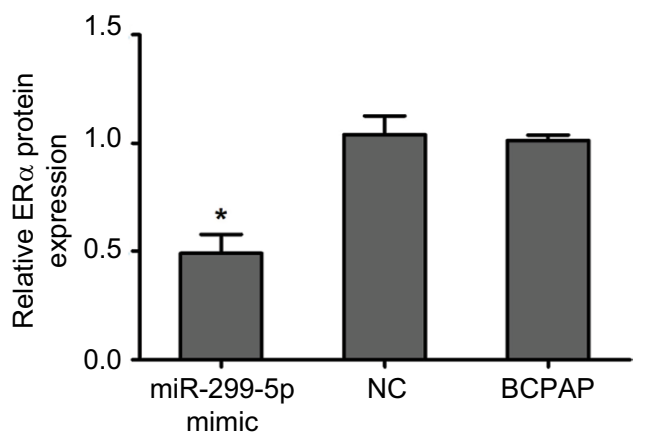

B

Position 4206-4212 of ESR1 3' UTR

ESR1 3'UTR-Wt...UAAUGAACACACUUGUAAACCU... miT-299-5p

UACAUACACCCUGCCAUUUGGU

ESR1 3'UTR-Mt...UAAUGAAACACACUUCAUUUGGU...

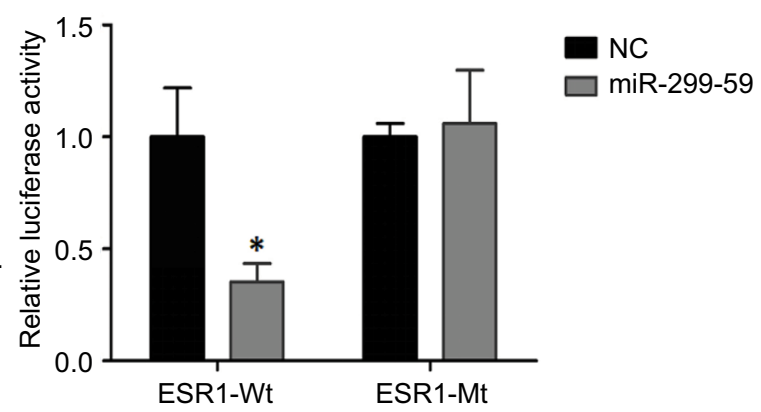

Figure $3 E R \alpha$ was a direct target of miR-299-5p.

Notes: (A) Relative mRNA expression level of ESRI was detected by qRT-PCR, and protein expression level of ER $\alpha$ was detected using Western blotting in PTC cells transfected with miR-299-5p mimic or NC. Data are presented as the mean $\pm S D$, analyzed using independent samples $t$-test. $* P<0.05$ vs NC. (B) The predicted miR-299-5p binding sites in ESR-I-3'-UTR-Wt and the designed mutant sequence (ESR-I-3'-UTR-Mt) are indicated, and a luciferase reporter assay was conducted. Data are presented as the mean \pm SD. $* P<0.05$ vs ESR-I-3-3'-UTR-Wt+NC. ESR-I-3'-UTR-Wt, 3'-UTR region of ESRI

Abbreviations: $E R \alpha$, estrogen receptor $\alpha$; NC, negative control; qRT-PCR, quantitative real-time reverse transcription PCR.

miR-299-5p might inhibit the migration and invasion of PTC cells by targeting ER $\alpha$ to affect the expression of Gli1. To confirm our hypothesis, we first detected whether miR-299-5p could also regulate the protein level of Gli1. The results indicated that overexpression of miR-299-5p reduced the protein level of Gli1. Next, Western blotting results indicated that overexpression of ER $\alpha$ significantly rescued the inhibition of Gli1 expression in the presence of miR-299-5p mimic (Figure 6D). In addition, BCPAP cell lysates were coimmunoprecipitated with anti-ER $\alpha$ antibodies, followed by Western blotting assay with antiGli1 antibodies. A $120 \mathrm{kDa}$ band corresponding to Gli1 was found in the input group, and the group coimmunoprecipitated with anti-ER $\alpha$ antibodies (Figure 6E). Our results revealed that Gli1 is a direct client protein of ER $\alpha$ and that miR-299-5p might inhibit migration and invasion by regulating the $\mathrm{ER} \alpha / \mathrm{Glil}$ complex.

\section{Discussion}

In a previous study, a microarray identified that miR-299-5p was significantly downregulated in extrathyroid invasion PTC tissues, indicating that miR-299-5p could play important regulatory roles in PTC invasion. In this study, we found that the expression of miR-299-5p was significantly lower in 109 PTC tissues compared with paired adjacent normal thyroid tissues. The low expression of miR-299-5p correlated with gender and ETE. In addition, overexpression of miR-299-5p inhibited ER $\alpha$ expression, thereby suppressing migration and invasion of BCPAP cells. Collectively, our findings suggest that miR-299-5p acts as a PTC suppressor. 
A
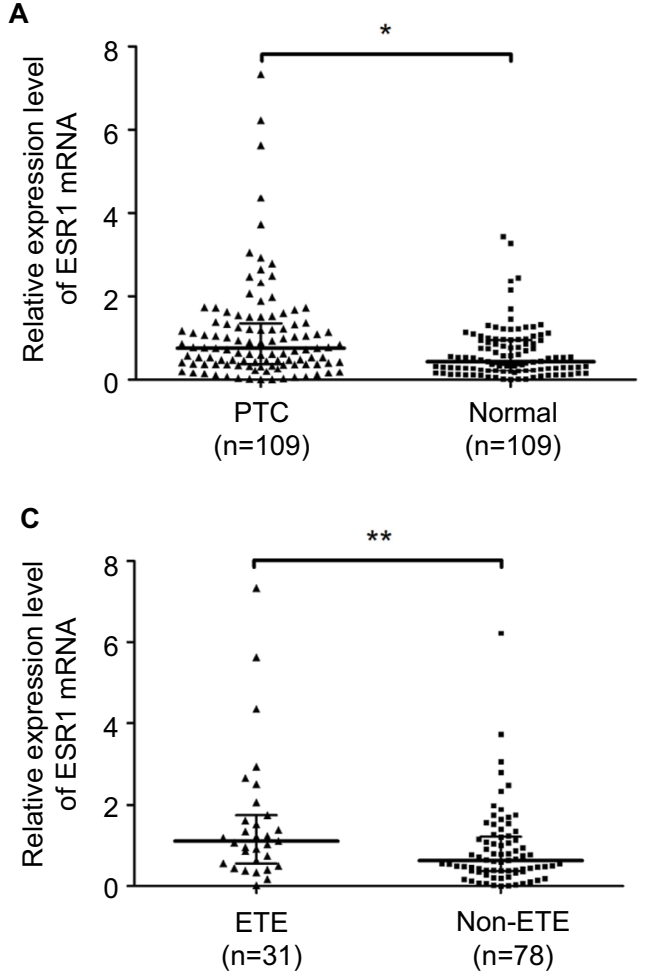

B

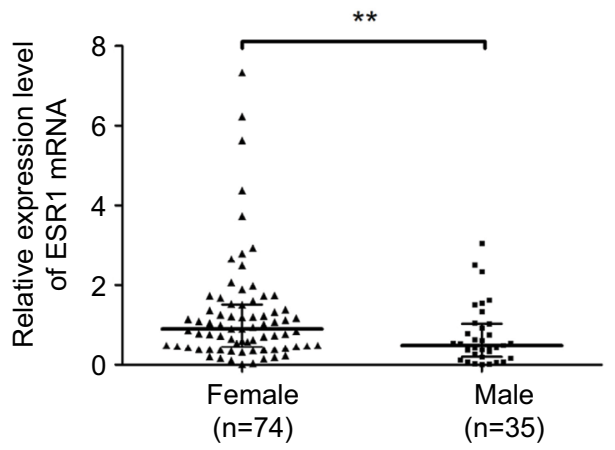

D

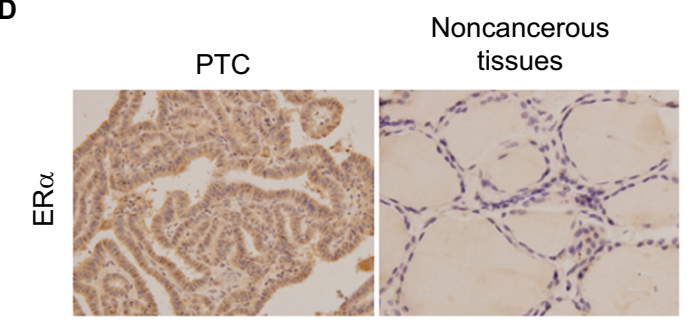

$\mathbf{F}$
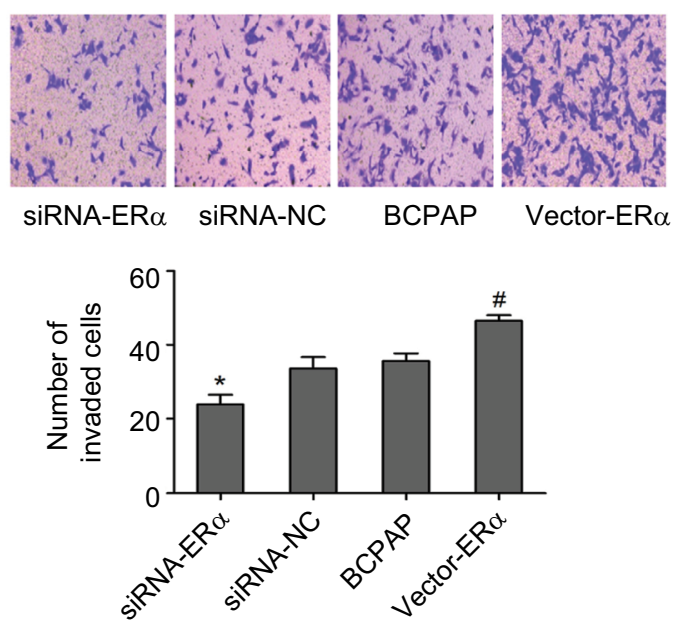

Figure 4 ER $\alpha$ was overexpressed and associated with migration and invasion in PTC.

Notes: (A) The relative mRNA expression level of ESRI in 109 PTC tissues and paired adjacent normal thyroid tissues was determined using qRT-PCR. (B) The relative mRNA expression level of ESRI in 74 female and 35 male PTC tissues was determined using qRT-PCR. (C) The relative mRNA expression level of ESRI in 3 I ETE and 78 non-ETE PTC tissues was determined using qRT-PCR. (D) The protein expression level of ER $\alpha$ was detected using IHC in PTC tissues and adjacent normal thyroid tissues. (E) The protein expression of ER $\alpha$ was detected using Western blotting in PTC tissues and adjacent normal thyroid tissues. (F) Transwell assays were used to evaluate the invasion capacity after upregulating and downregulating ER $\alpha$ levels in PTC cells. Data are presented as the mean \pm SD. $* P<0.05$, $* * P<0.01$ vs NC. $\# P<0.05$ vs NC.

Abbreviations: ER $\alpha$, estrogen receptor $\alpha$; ETE, extrathyroidal extension; IHC, immunohistochemistry; NC, negative control; PTC, papillary thyroid cancer; qRT-PCR, quantitative real-time reverse transcription PCR.

The anticancer role of miR-299-5p has been reported in other cancer types, such as breast cancer, hepatocellular cancer, and colorectal cancer. ${ }^{7,8,10}$ Shevde et $\mathrm{al}^{7}$ reported that miR-299-5p regulates osteopontin (OPN) and that increasing the expression of OPN via decreased levels of miR-299-5p plays an important role in enhancing the proliferation of breast cancer cells. Jin et $a l^{8}$ demonstrated that miR-299-5p could act as a tumor suppressor to mediate the antitumor mechanism of OSW-1 (a saponin with marked antitumor activities) in hepatocellular cancer. In addition, Fateh et a $1^{10}$ showed that the expression level of miR-299-5p was significantly downregulated in a group of colorectal cancer tissues and could be used as a predictive biomarker in the diagnosis of colorectal cancer. In our study, the different expression 
A

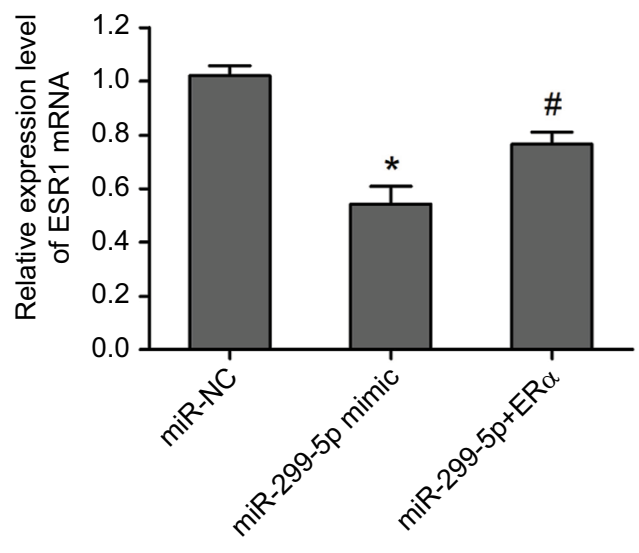

B

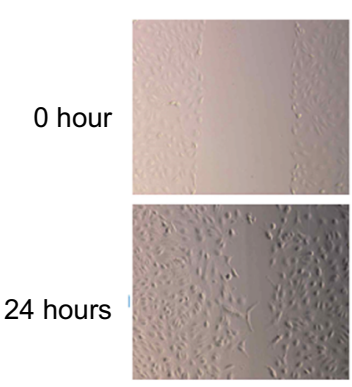

miR-NC

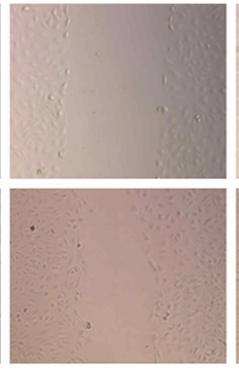

miR-299-5p mimic

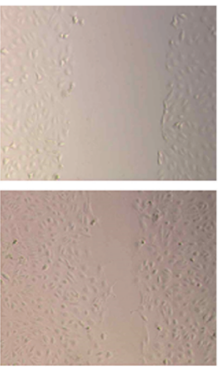

miR-299-5p
C

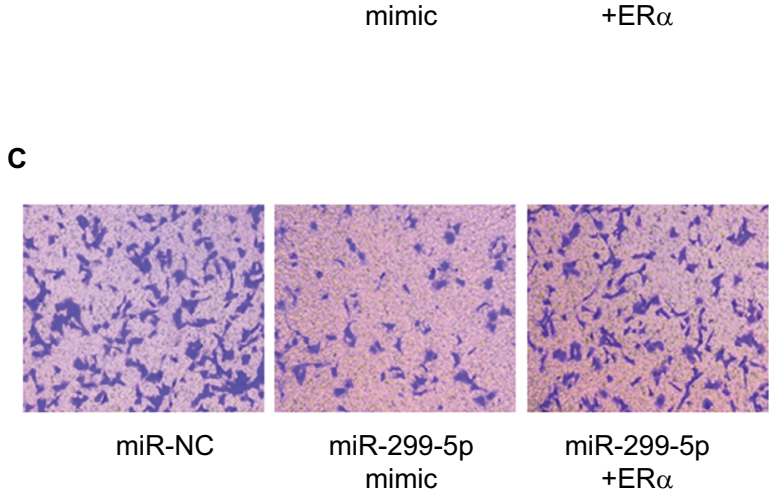

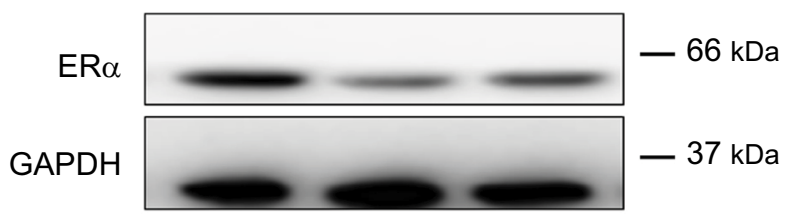

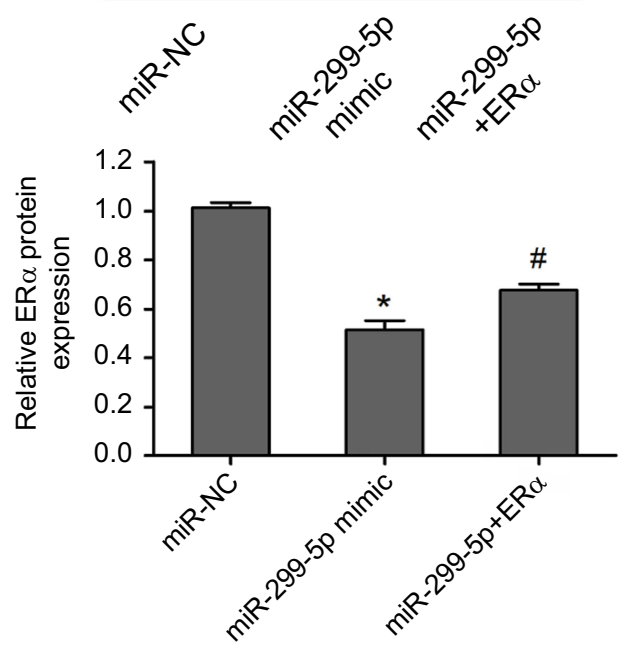

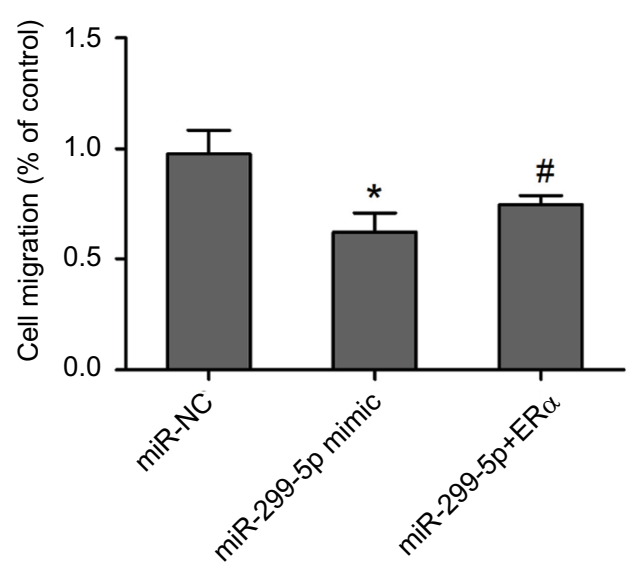

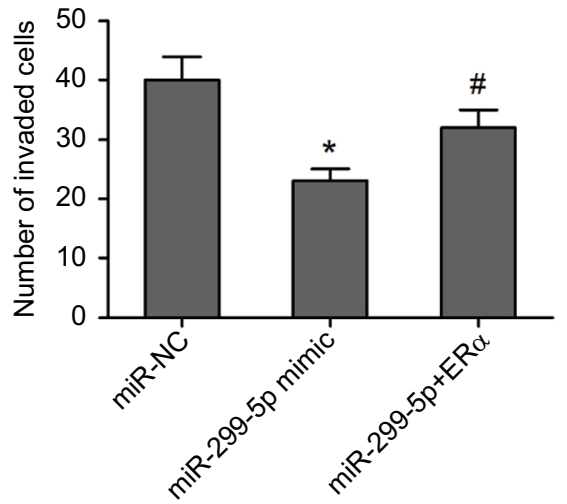

Figure 5 Overexpression of ER $\alpha$ could partly impair miR-299-5p induced inhibition of malignant and invasion behavior in PTC cells.

Notes: (A) Relative mRNA expression level of ESRI was detected using qRT-PCR, and the protein expression level of ER $\alpha$ was detected using Western blotting in PTC cells transfected with miR-299-5p mimic, miR-299-5p+ER $\alpha$, or NC. (B) A wound healing assay was applied to analyze the migration capacity in PTC cells after transfection with miR-299-5p mimic, miR-299-5p+ER $\alpha$, or NC. (C) Transwell assays were used to evaluate the invasion in PTC cells after transfection with miR-299-5p mimic, miR-299$5 p+E R \alpha$, or NC. Data are presented as the mean \pm SD. ${ }^{*} P<0.05$ vs NC, ${ }^{*} P<0.05$ vs miR-299-5 $+E R \alpha$.

Abbreviations: $\mathrm{ER} \alpha$, estrogen receptor $\alpha$; NC, negative control; PTC, papillary thyroid cancer; qRT-PCR, quantitative real-time reverse transcription PCR. 
A

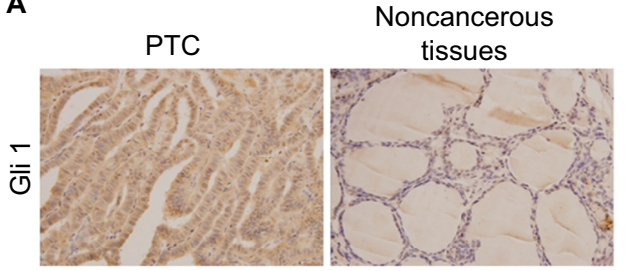

C
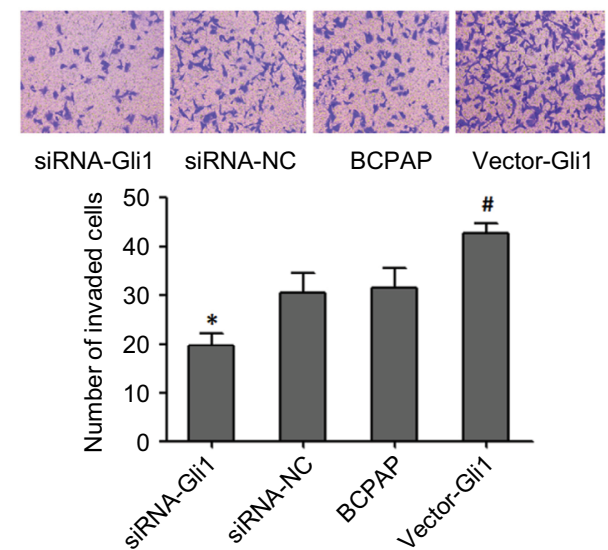

E

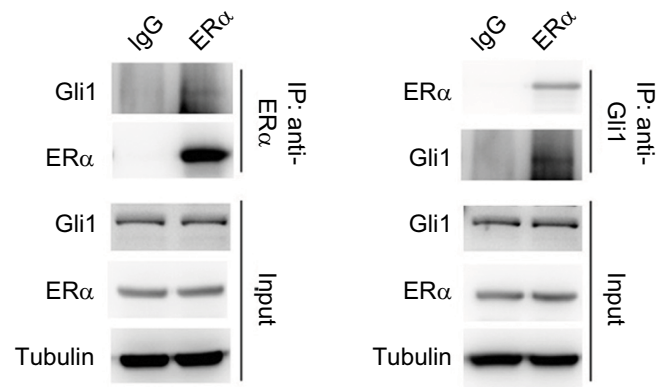

B

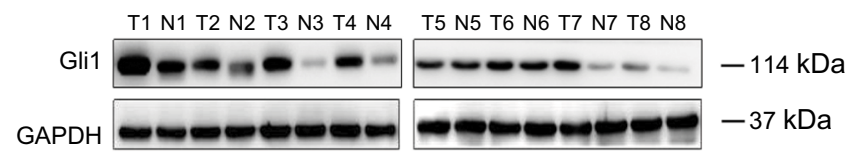

D
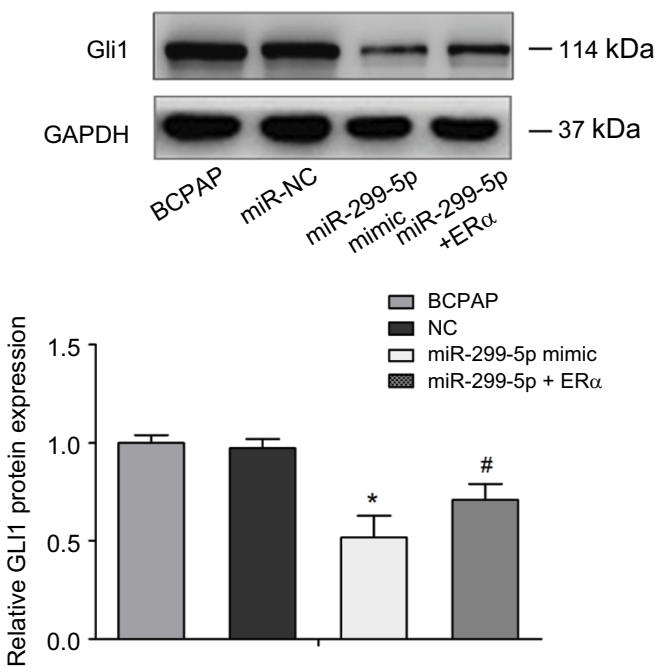

F

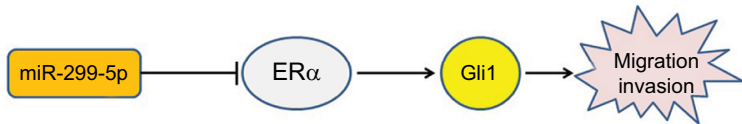

Figure 6 miR-299-5p regulates migration and invasion through ER $\alpha /$ Gli I complex.

Notes: (A) The protein expression level of Glil was detected using IHC in PTC tissues and adjacent normal thyroid tissues. (B) The protein expression level of Glil was detected using Western blotting in PTC tissues and adjacent normal thyroid tissues. (C) The invasion capacity after upregulating and downregulating Glil in PTC cells were evaluated using Transwell assays. (D) The protein expression level of Glil was detected using Western blotting in PTC cells transfected with miR-299-5p mimic, miR-299$5 p+E R \alpha$, or NC. Data are presented as the mean \pm SD. $* P<0.05$ vs NC, ${ }^{*} P<0.05$ vs miR-299-5p+ER $\alpha$. (E) BCPAP cell lysates were coimmunoprecipitated with ER $\alpha$ antibody and followed by Western blotting assay with anti-Glil antibodies. (F) Schematic of the proposed mechanism of miR-299-5p in PTC. In ER $\alpha$-positive cell lines, miR-299-5p may inhibit the migration and invasion of PTC cells by targeting ER $\alpha$ to affect the expression of Glil.

Abbreviations: $\mathrm{ER} \alpha$, estrogen receptor $\alpha$; Gli I, GLI family zinc finger I; IHC, immunohistochemistry; NC, negative control; PTC, papillary thyroid cancer.

of miR-299-5p in men and women, and the invasion-suppressive role of miR-299-5p by targeting ER $\alpha$, indicated the importance of this essential pathway in PTC.

Estrogens are involved in the growth and differentiation of the normal mammary gland, and the biological effect of estrogen is mediated by several receptors, including $\mathrm{ER} \alpha$ and ER $\beta$. ER $\alpha$ contributes to the development of gynecological tumors, especially breast cancer. In PTC, overexpression of ER $\alpha$ could modulate MMP-2 and MMP-9 secretion and activity in thyroid cancer cells. Loss of the ER by siRNA transfection resulted in loss of estradiol-mediated migration and invasion of thyroid cancer cells. ${ }^{13}$ In the present study, we demonstrated the invasion suppression role of miR-299-5 $\mathrm{p}$ by functional experiments and identified the miR-299-5pbinding site of ESR 1 using a luciferase reporter gene assay.

To further determine the potential molecular mechanism of miR-299-5p in regulating ER $\alpha$, we focused on the downstream proteins that interact with ER $\alpha$. The level of Gli1, the key regulator in the Shh pathway, has been reported to correlate with the invasiveness in many different cancers. ${ }^{16}$ Our previous study reported that Gli1 was significantly overexpressed and correlated with larger tumor size, the presence of ETE, lymph nodes metastasis, and higher TNM stage in PTC. ${ }^{19}$ In breast cancer, Gli1 may be responsible 
for the estrogen-induced epithelial-esenchymal transition (EMT) in ER-positive breast cancer cells..$^{20,21} \mathrm{E} 2$ induces transcriptional activation of Gli1 probably by enriching ER occupancy at the Glil promoter. Sun et $\mathrm{al}^{20}$ reported that ER expression positively correlated with Gli1 levels. Estrogen may promote ER-positive breast cancer cell development and EMT via Gli1. Our results indicated that overexpression of miR-299-5p reduced the protein level of Gli1 in BCPAP cells. Moreover, a coimmunoprecipitation assay revealed the interaction between ER $\alpha$ and Gli1, which increased our understanding of the molecular interactions between these two pathways.

Our study highlighted the oncotarget potential of miR299-5p expression in ER $\alpha$-positive PTC BCPAP cells. Moreover, this is the first study to describe the relationships among miRNAs and the ER and Shh pathways, which might encourage further exploration of the anticancer function of miR-299-5p, although our results need to be validated in a larger sample cohort.

\section{Conclusion}

This study revealed that miR-299-5p is significantly downregulated and has clinical significance in PTC migration and invasion. ER $\alpha$ and the ER $\alpha /$ Gli1 1 complexes were demonstrated to be the direct binding target and functional target of miR-299-5p, respectively. Our data suggested that miR-299-5p could participate in PTC migration and invasion and could be a potential therapeutic target for patients with aggressive PTC tumors (Figure 6F).

\section{Acknowledgments}

This research was supported by National Natural Science Foundation of China (grant number: 81502319), the Liaoning Province PhD Start-up Fund (grant number: 201501008), the Liaoning BaiQianWan Talents Program (grant number: 2014921033), the Science and Technology Project of Shenyang City (grant number: F16-205-1-41), and Natural Science Foundation of Liaoning Province (grant number: 2015020536).

\section{Disclosure}

The authors report no conflicts of interest in this work.

\section{References}

1. Mao Y, Xing M. Recent incidences and differential trends of thyroid cancer in the USA. Endocr Relat Cancer. 2016;23(4):313-322.

2. Kunavisarut T. Diagnostic biomarkers of differentiated thyroid cancer. Endocrine. 2013;44(3):616-622.
3. Lundgren CI, Hall P, Dickman PW, Zedenius J. Clinically significant prognostic factors for differentiated thyroid carcinoma: a populationbased, nested case-control study. Cancer. 2006;106(3):524-531.

4. Falcone G, Felsani A, D’Agnano I. Signaling by exosomal microRNAs in cancer. J Exp Clin Cancer Res. 2015;34:32.

5. Esquela-Kerscher A, Slack FJ. Oncomirs - microRNAs with a role in cancer. Nat Rev Cancer. 2006;6(4):259-269.

6. Wang Z, Zhang H, He L, et al. Association between the expression of four upregulated miRNAs and extrathyroidal invasion in papillary thyroid carcinoma. Onco Targets Ther. 2013;6:281-287.

7. Shevde LA, Metge BJ, Mitra A, et al. Spheroid-forming subpopulation of breast cancer cells demonstrates vasculogenic mimicry via hsa-miR299-5p regulated de novo expression of osteopontin. J Cell Mol Med. 2010;14(6B):1693-1706.

8. Jin JC, Jin XL, Zhang X, Piao YS, Liu SP. Effect of OSW-1 on microRNA expression profiles of hepatoma cells and functions of novel microRNAs. Mol Med Rep. 2013;7(6):1831-1837.

9. Formosa A, Markert EK, Lena AM, et al. MicroRNAs, miR-154, miR-299-5p, miR-376a, miR-376c, miR-377, miR-381, miR-487b, miR-485-3p, miR-495 and miR-654-3p, mapped to the 14q32.31 locus, regulate proliferation, apoptosis, migration and invasion in metastatic prostate cancer cells. Oncogene. 2014;33(44):5173-5182.

10. Fateh A, Feizi MAH, Safaralizadeh R, Azarbarzin S. Importance of miR-299-5p in colorectal cancer. Ann Gastroenterol. 2017;30(3): 322-326.

11. Rahbari R, Zhang L, Kebebew E. Thyroid cancer gender disparity. Future Oncol. 2010;6(11):1771-1779.

12. Fan D, Liu SY, van Hasselt CA, et al. Estrogen receptor $\alpha$ induces prosurvival autophagy in papillary thyroid cancer via stimulating reactive oxygen species and extracellular signal regulated kinases. J Clin Endocrinol Metab. 2015;100(4):E561-E571.

13. Rajoria S, Suriano R, George A, et al. Estrogen induced metastatic modulators MMP-2 and MMP-9 are targets of 3,3'-diindolylmethane in thyroid cancer. PLoS One. 2011;6(1):e15879.

14. Dong W, Zhang H, Li J, et al. Estrogen Induces Metastatic Potential of Papillary Thyroid Cancer Cells through Estrogen Receptor $\alpha$ and $\beta$. Int J Endocrinol. 2013;2013:941568.

15. Huang Y, Dong W, Li J, Zhang H, Shan Z, Teng W. Differential expression patterns and clinical significance of estrogen receptor- $\alpha$ and $\beta$ in papillary thyroid carcinoma. BMC Cancer. 2014;14:383.

16. Beachy PA, Hymowitz SG, Lazarus RA, Leahy DJ, Siebold C. Interactions between Hedgehog proteins and their binding partners come into view. Genes Dev. 2010;24(18):2001-2012.

17. Ke Z, Caiping S, Qing Z, Xiaojing W. Sonic hedgehog-Gli1 signals promote epithelial-mesenchymal transition in ovarian cancer by mediating PI3K/AKT pathway. Med Oncol. 2015;32(1):368.

18. Thomas ZI, Gibson W, Sexton JZ, et al. Targeting GLI1 expression in human inflammatory breast cancer cells enhances apoptosis and attenuates migration. Br J Cancer. 2011;104(10):1575-1586.

19. Dong W, Cui J, Tian X, et al. Aberrant sonic hedgehog signaling pathway and STAT3 activation in papillary thyroid cancer. Int J Clin Exp Med. 2014;7(7):1786-1793.

20. Sun Y, Wang Y, Fan C, et al. Estrogen promotes stemness and invasiveness of ER-positive breast cancer cells through Gli1 activation. Mol Cancer. 2014;13:137.

21. Xu L, Kwon YJ, Frolova N, et al. Gli1 promotes cell survival and is predictive of a poor outcome in ERalpha-negative breast cancer. Breast Cancer Res Treat. 2010;123(1):59-71.

22. Mete O, Rotstein L, Asa SL. Controversies in thyroid pathology: thyroid capsule invasion and extrathyroidal extension. Ann Surg Oncol. 2010;17(2):386-391.

23. Rajoria S, Suriano R, Shanmugam A, et al. Metastatic phenotype is regulated by estrogen in thyroid cells. Thyroid. 2010;20(1):33-41.

24. Kumar A, Klinge CM, Goldstein RE. Estradiol-induced proliferation of papillary and follicular thyroid cancer cells is mediated by estrogen receptors alpha and beta. Int J Oncol. 2010;36(5):1067-1080. 


\section{Supplementary materials}

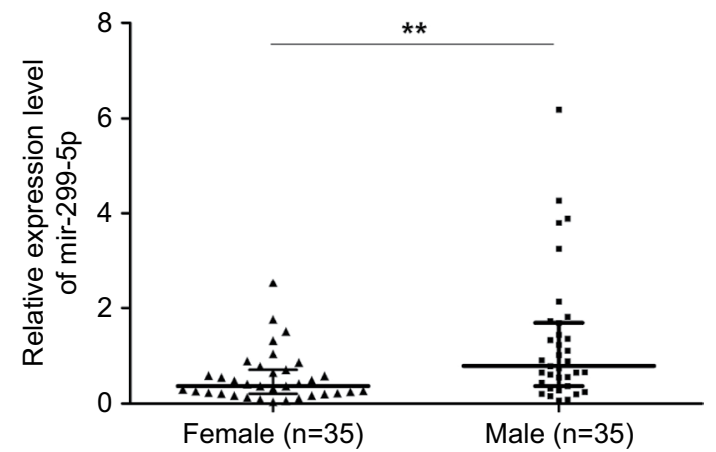

Figure SI miR-299-5p expression between male $(n=35)$ and female $(n=35)$ patients. Note: $* * P<0.01$.

A

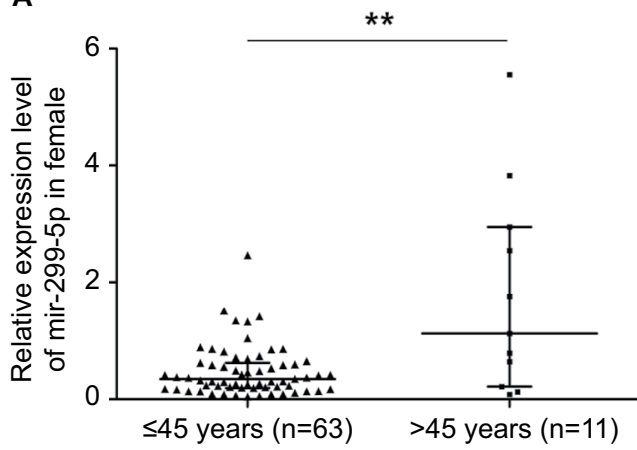

B

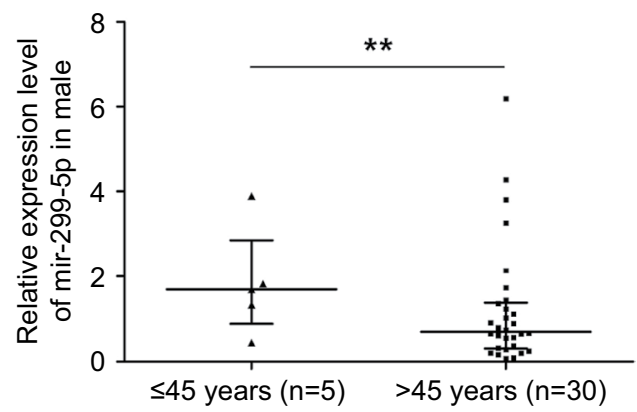

Figure S2 miR-299-5p expression in male and female patients in different age subgroups.

Notes: (A) miR-299-5p expression between reproductive age ( $\leq 45$ years) and advanced reproductive age ( $>45$ years) subgroups in male patients. (B) miR-299-5p expression between reproductive age ( $\leq 45$ years) and advanced reproductive age ( $>45$ years) subgroups in female patients. ${ }^{*} * P<0.01$. 

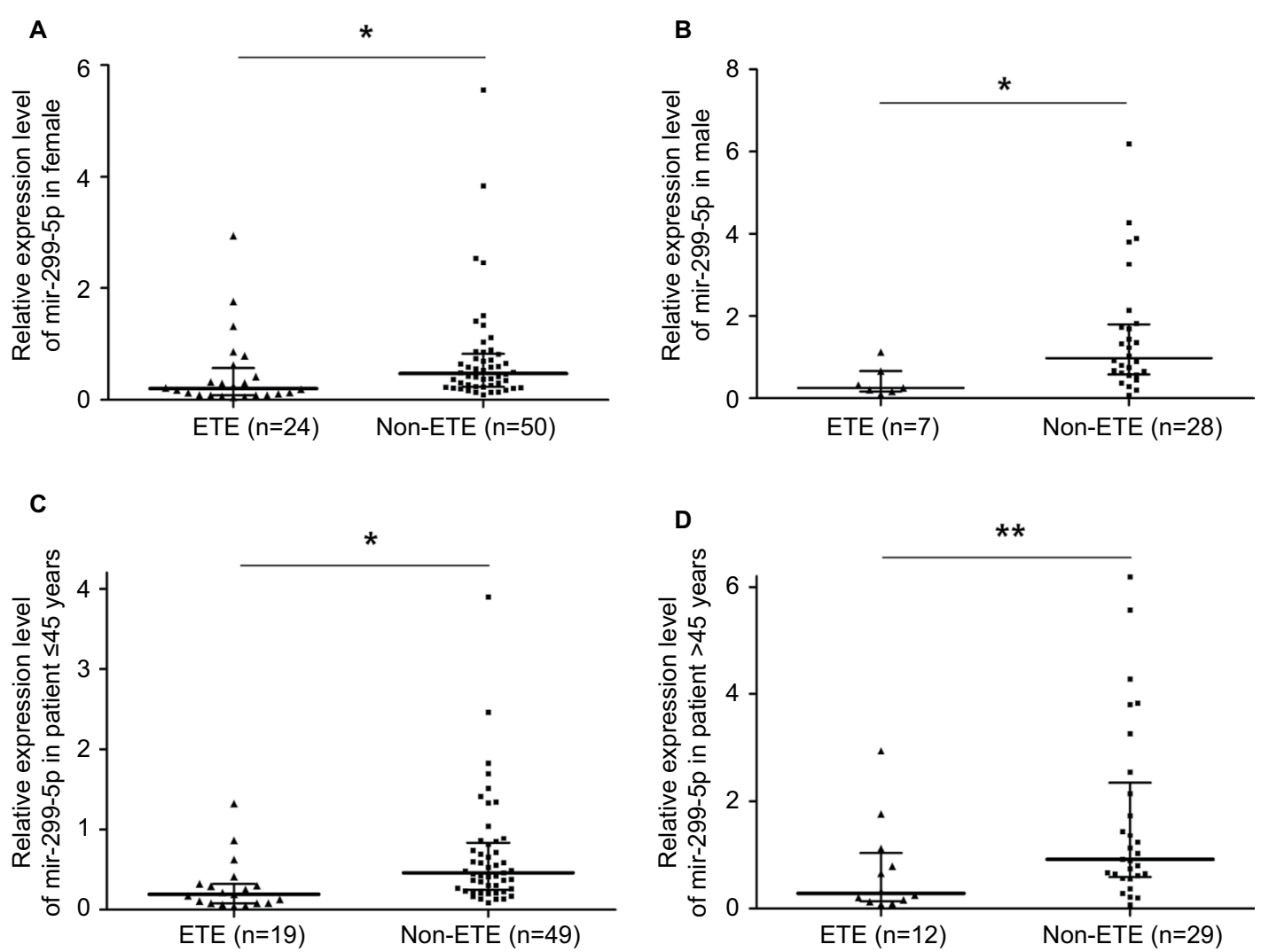

Figure S3 miR-299-5p expression in ETE vs non-ETE patients in gender-specific and age-specific manners.

Notes: (A) miR-299-5p expression between ETE and non-ETE subgroups in female patients. (B) miR-299-5p expression between ETE and non-ETE subgroups in male patients. (C) miR-299-5p expression between ETE and non-ETE subgroups in reproductive age ( $\leq 45$ years) patients. (D) miR-299-5p expression between ETE and non-ETE subgroups in advanced reproductive age ( $>45$ years) patients. $* P<0.05$ and $* * P<0.0$ I.

Abbreviation: ETE, extrathyroidal extension.

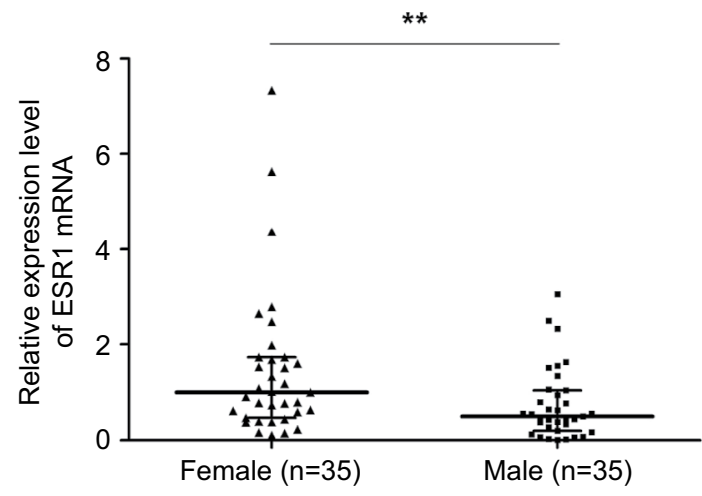

Figure S4 ESRI expression between male $(n=35)$ and female $(n=35)$ patients.

Note: $* * P<0.01$.

Cancer Management and Research

\section{Publish your work in this journal}

Cancer Management and Research is an international, peer-reviewed open access journal focusing on cancer research and the optimal use of preventative and integrated treatment interventions to achieve improved outcomes, enhanced survival and quality of life for the cancer patient. The manuscript management system is completely online and includes

a very quick and fair peer-review system, which is all easy to use. Visit $\mathrm{http}: / / \mathrm{www}$.dovepress.com/testimonials.php to read real quotes from published authors. 